\title{
Research Microscopy-assisted Cytomorphometric Analysis of Oral Exfoliated Cells in Leukoplakia and OSCC: A Comparative Study
}

\author{
Preethi Sharma ${ }^{1}$, Minal Chaudhary ${ }^{2}$, Rajul Ranka ${ }^{3}$
}

\begin{abstract}
Aim: To analyze and compare the cytomorphometric variations of oral exfoliated cells in normal healthy subjects, leukoplakia, and oral squamous cell carcinoma (OSCC) using LEICA DMLB2 research microscope.

Materials and methods: Papanicolaou staining (PAP) was performed on the oral smears obtained from the study subjects. The study group consisted of normal healthy subjects (group I), leukoplakia (group II), and OSCC (group III). Photomicrographs of randomly selected 100 cells from each smear were captured at 40× magnification using a research microscope to obtain cell diameter (CD), nuclear diameter (ND), cellular area (CA), nuclear area (NA) and, nuclear-to-cytoplasmic (N/C) ratio. Descriptive and inferential statistics were used in the statistical analysis of the values obtained using one-way analysis of variance (ANOVA) and multiple comparison Tukey test. SPSS 22.0 version and GraphPad Prism 7.0 version software were used in the analysis, and the level of significance $p<0.05$ was considered.

Results: The results of this study demonstrated a statistically significant increase in ND, NA with a decrease in CD, CA, and altered N/C ratio in leukoplakia and OSCC when compared to normal healthy subjects.

Conclusion: Oral exfoliative cytology using a commercially available toothbrush is a simple, non-invasive, relatively quick, and inexpensive technique that could be used in the screening of suspicious oral potentially malignant disorders (PMDs) and OSCC. Quantitative parameters, such as, morphometry, are reproducible and eliminate the observer bias as it is obtained by software analysis and hence improves the accurateness in the diagnosis.

Clinical significance: Brush biopsy can also be used in any setup as well as a mass screening of dysplasias, PMDs, and early carcinomas in countries like India which holds the major core of the disease constraint.

Keywords: Cytology, Dysplasia, Leukoplakia, Oral cancer.

World Journal of Dentistry (2021): 10.5005/jp-journals-10015-1794
\end{abstract}

\section{INTRODUCTION}

A topographical variation exists in the incidence of head and neck cancer among different nations of the world and various states of the country. Worldwide over 175,000 cases are diagnosed every year. ${ }^{1}$ Oral cancer reports for about $40 \%$ of all cancers in the Indian subcontinent. ${ }^{2}$ It is the most common cause of morbidity regardless of the various advancements made in the treatment plan.

Oral cancer in the early phase may implicate itself and appear as a benign and asymptomatic lesion. The unawareness of the lesion is the main factor behind the tumor proceeding into an advanced stage. An approach to forbid this problem would be earlier detection of potentially malignant disorders (PMDs) such as leukoplakia, oral submucous fibrosis, erythroplakia, and oral lichen planus. $^{3-7}$ Exfoliative cytology is a simpler, painless diagnostic technique by which earlier detection of the lesion can be made. The application of this method was limited due to the subjective nature of its interpretation and highly false-negative results. Quantitative methods, such as, image analysis systems were introduced to overcome these limitations. The application of morphometry makes it easier to analyze variations in size, shape, and staining intensity of the cell and nuclei. ${ }^{8}$ There has been increasing applications of research microscope in various universities and medical laboratories in the last few years. Research microscope has the advantage of providing high-resolution images and the images can be saved in the virtual archives. It allows telepathology, storage of archives of histopathology in hospital settings, research studies, case study
${ }^{1-3}$ Department of Oral Pathology, Sharad Pawar Dental College and Hospital, Datta Meghe Institute of Medical Sciences, Sawangi (Meghe), Wardha, Maharashtra, India

Corresponding Author: Preethi Sharma, Department of Oral Pathology, Sharad Pawar Dental College and Hospital, Datta Meghe Institute of Medical Sciences, Sawangi (Meghe), Wardha, Maharashtra, India, Phone: +91 7020536570, e-mail: dr.preethisharma@gmail.com

How to cite this article: Sharma P, Chaudhary M, Ranka R. Research Microscopy-Assisted Cytomorphometric Analysis of Oral Exfoliated Cells in Leukoplakia and OSCC: A Comparative Study. World J Dent 2021;12(1):17-21.

Source of support: Nil

Conflict of interest: None

libraries, and e-learning. Its application is convenient for both researchers and professionals. The diagnostic criteria for PMDs are based on nuclear and cellular changes favoring cytopathologic diagnosis. The aim and the objectives of the study were to analyze the cytomorphometric variations, such as, cellular and nuclear diameter (ND), cellular and nuclear area (NA), nuclear-cytoplasmic ratio (N/C) in the cytological smears of normal healthy subjects, leukoplakia, oral squamous cell carcinoma (OSCC), and also to compare cellular and ND, cellular and NA, nuclear-cytoplasmic ratio $(\mathrm{N} / \mathrm{C})$ in normal, leukoplakia, and OSCC. 


\section{Materials and Methods}

The study was carried out in the Department of Oral Pathology and Microbiology, Sharad Pawar Dental College and Hospital, Sawangi, Wardha. The subjects attending the outpatient department were considered for the study through a convenience sampling method. The study included 50 normal healthy subjects (group I) who came to OPD were taken as control, 50 patients clinically diagnosed with leukoplakia (group II), and 50 patients clinically diagnosed with OSCC (group III). Patients with systemic illness, patients undergoing treatment for leukoplakia and OSCC, patients with recurrence of oral cancer were excluded from the study. The complete procedure was explained to the patients and informed consent was collected from all the study participants.

The clearance from the institutional ethical committee was obtained to undertake the study. Detailed information regarding personal details, history of any adverse habit was recorded. A detailed intraoral and extraoral examination along with a general medical examination was carried out in all subjects. Exfoliative cytology was performed using a commercially available toothbrush followed by a biopsy. Before the start of the procedure, the subjects were allowed to rinse their mouth with water to clear off the detritus, and the area of suspicion was cleaned with a swab. The toothbrush was sterilized for 24 hours in chlorhexidine gluconate $(0.2 \%)$ mouthwash and was disposed of after every use. In patients with clinically diagnosed leukoplakia, the smear was obtained from a thick keratotic area. In patients with clinically diagnosed OSCC, the smear was obtained from the ulcerated area and in normal healthy subjects, the smear was taken from the normal-appearing buccal mucosa. The selected areas were brushed repeatedly applying moderate pressure in one direction till the appearance of pinpoint bleeding areas. The material from the brush was evenly spread on the full length of the dried glass slide. The smear was obtained on 2-3 slides for each patient and only one slide having a larger number of cells was analyzed. Thus, a total of 150 Papanicolaou staining (PAP) stained slides were analyzed. The slides were dipped immediately in 95\% isopropyl alcohol for proper fixation of cells. The staining of smears was performed by the PAP technique using a commercially available staining kit-Magic PAP stain kit, Pathozyme Diagnostics.

The cytomorphometric variations were analyzed under a high power field (40× magnification) on randomly selected 100 cells from each smear. To keep away from the repetition of the same cells, the stage of the microscope was moved in a step ladder pattern. The ND, cellular diameter, NA, and cellular area (CA) were measured after capturing the image on the LEICA DMLB2 Research microscope. The diameter and area of each cell and nucleus were obtained using the digitized cursor and the readings were obtained in the excel sheet form. The mean cellular diameter $(\mathrm{CD})$ and ND, CA and NA, nuclear-cytoplasmic ratio (N/C) were calculated in all three groups (Fig. 1).

\section{Results}

Descriptive and inferential statistics were used in the statistical analysis of the values obtained using the chi-square test, one-way analysis of variance (ANOVA), and multiple comparison Tukey test. SPSS 22.0 version and GraphPad Prism 7.0 version software were used in the analysis, and the level of significance $p<0.05$ was considered.

The mean ND (in $\mu \mathrm{m}$ ) in group I, group II, and group III were $114.08 \pm 4.63,142.11 \pm 6.93$, and $220.57 \pm 23.93$, respectively. Nuclear diameter was found to be significant among the groups when compared by the Chi-square test and one-way ANOVA (Fig. 2).

The mean cellular diameter (in $\mu \mathrm{m}$ ) in group I, group II, and group III were $761.49 \pm 34.07,485.69 \pm 13.98$, and $279.79 \pm 16.60$, respectively. The $C D$ was found to be significant among the groups when compared by the chi-square test and one-way ANOVA (Fig. 3).

The mean NA (in $\mu^{2}$ ) in group I, group II, and group III were $1646 \pm 247.03,3,150.39 \pm 355.89$, and 3,725.11 \pm 655.86 , respectively. The mean NA was highly significant among the groups when compared by the Chi-square test and one-way ANOVA (Fig. 4).

The mean CA (in $\mu \mathrm{m}^{2}$ ) in group I, group II, and group III were $296,184.33 \pm 45,476.50,141,983.21 \pm 21,675.45$, and 4,531.91 \pm 505.12 , respectively. The mean CA was highly significant among the groups when compared by the chi-square test and one-way ANOVA (Fig. 5).

The mean ND to cytoplasmic diameter ratio (in $\mu \mathrm{m}$ ) in group I, group II, and group III were $0.15 \pm 0.005,0.29 \pm 0.01$, and 0.78 \pm 0.05 , respectively. The ratio was found to be highly significant among the groups when compared by the chi-square test and one-way ANOVA (Fig. 6).

The mean NA to cytoplasmic area ratio (in $\mu \mathrm{m}^{2}$ ) in group I, group II, and group III were $0.006 \pm 0.007,0.02 \pm 0.003$, and 0.82 \pm 0.14 , respectively. The ratio was found to be highly significant among the groups when compared by one-way ANOVA (Fig. 7).
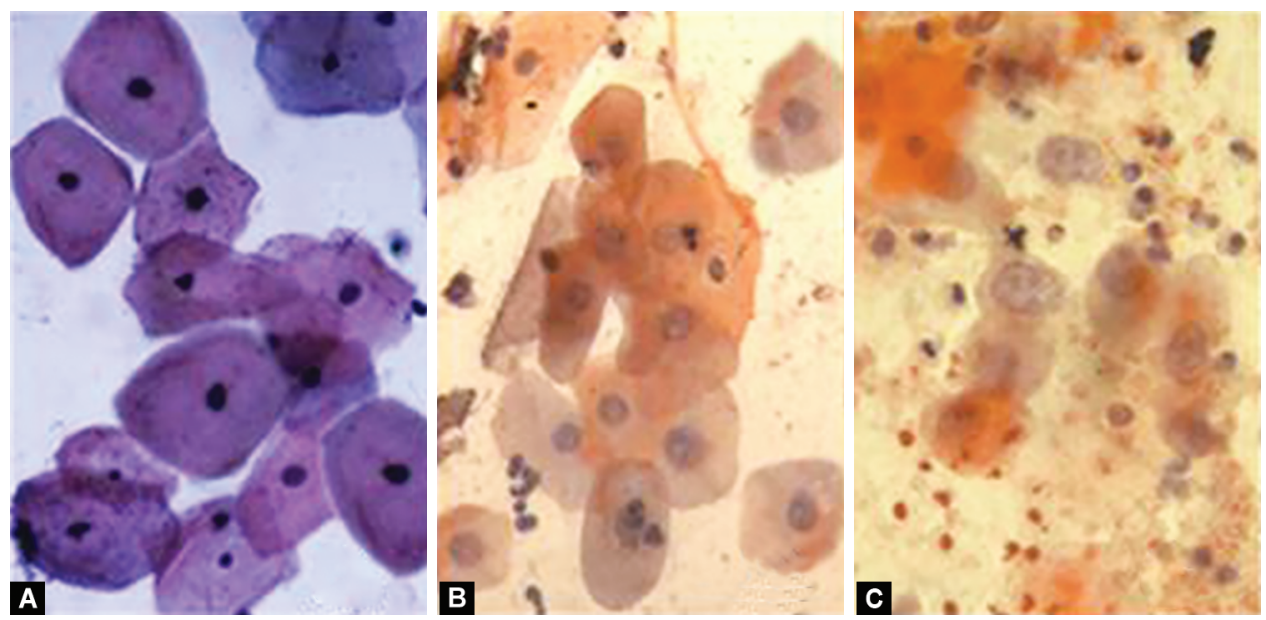

Figs 1A to C: (A) PAP stained smear of normal healthy subjects under 40x magnification; (B) PAP stained smear of leukoplakia under 40x magnification; (C) PAP stained smear of OSCC under $40 \times$ magnification 


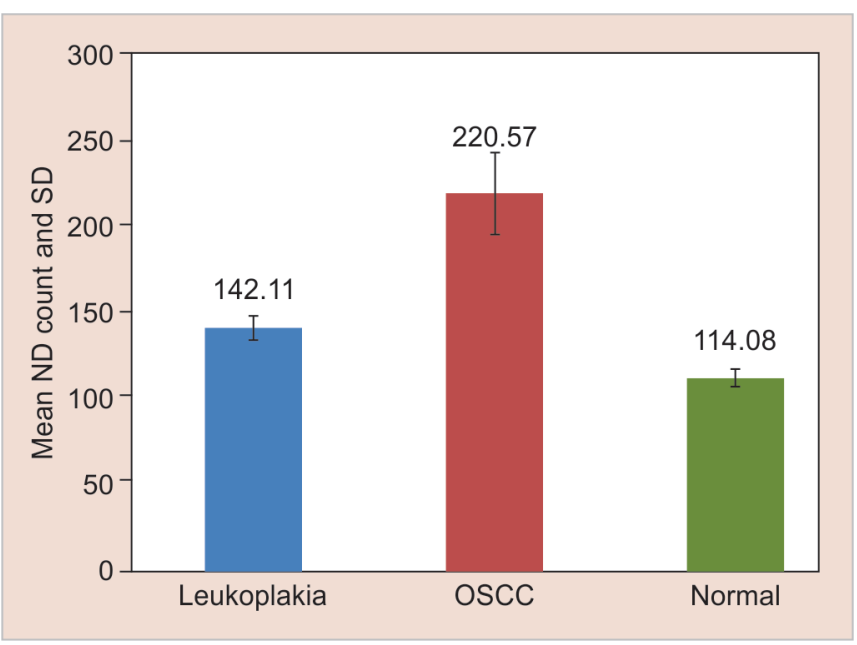

Fig. 2: Comparison of nuclear diameter in leukoplakia, OSCC, and normal healthy subjects

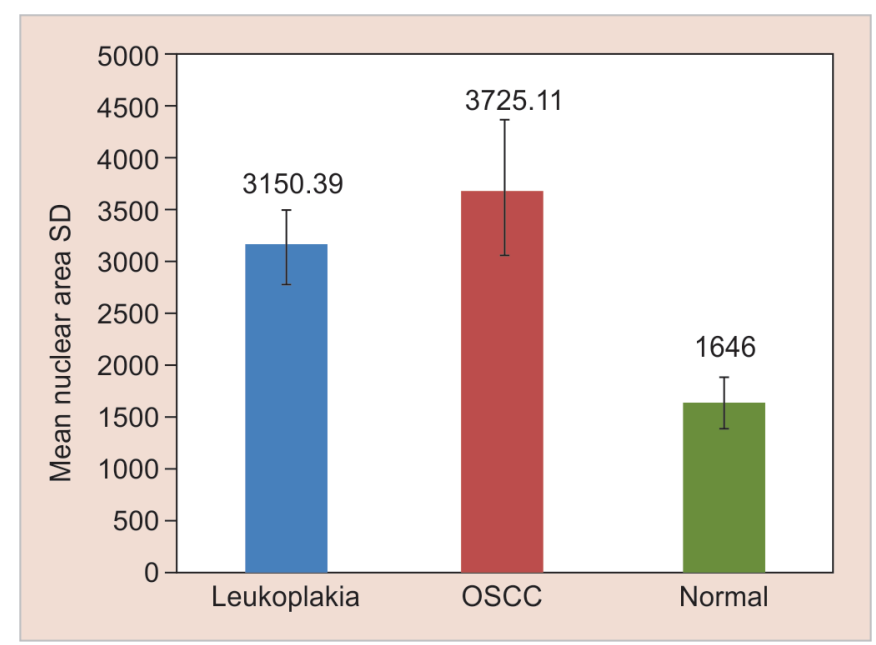

Fig. 4: Comparison of nuclear area in leukoplakia, OSCC, and normal healthy subjects

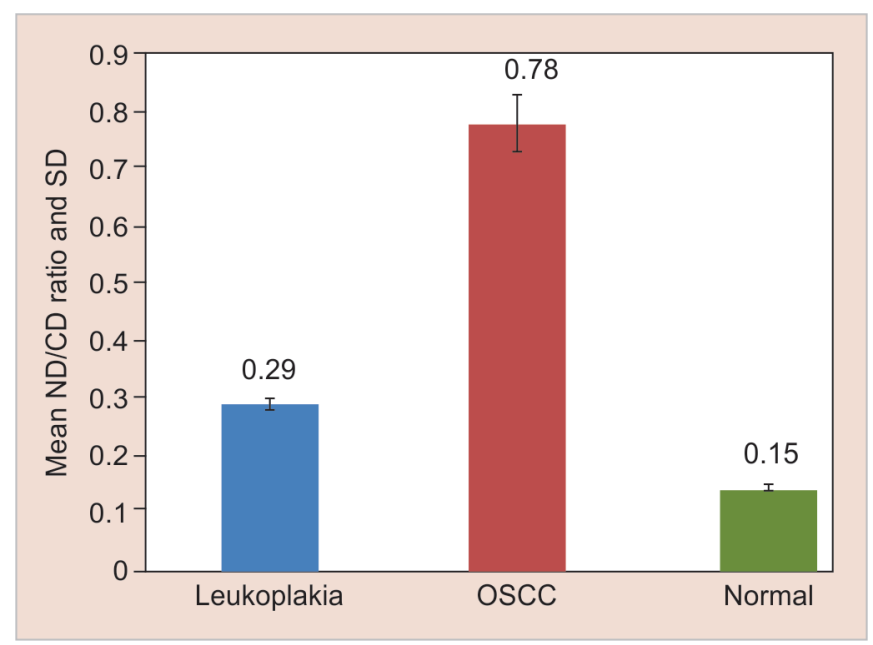

Fig. 6: Comparison of nuclear diameter and cellular diameter ratio in leukoplakia, OSCC, and normal healthy subjects

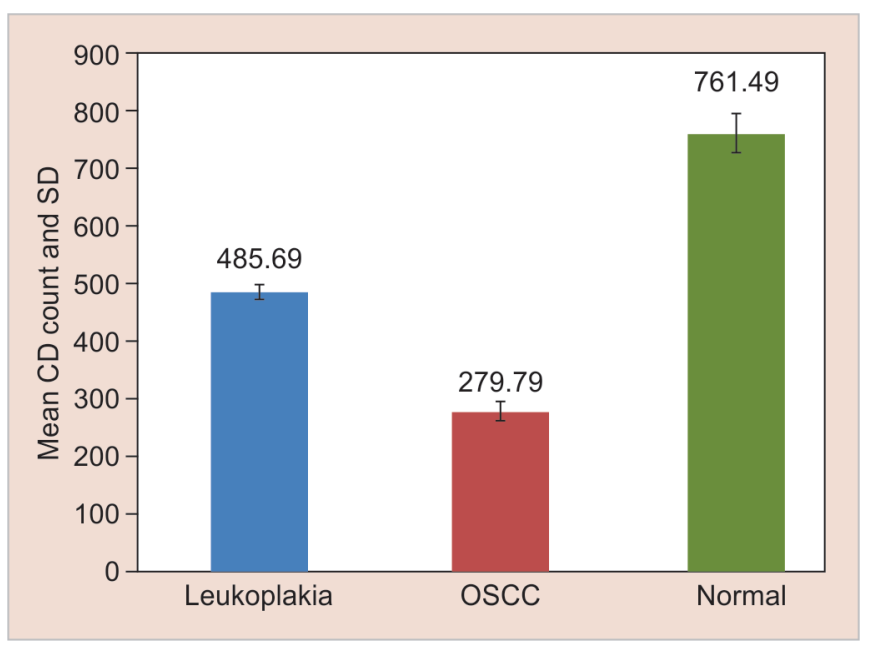

Fig. 3: Comparison of cellular diameter in leukoplakia, OSCC, and normal healthy subjects

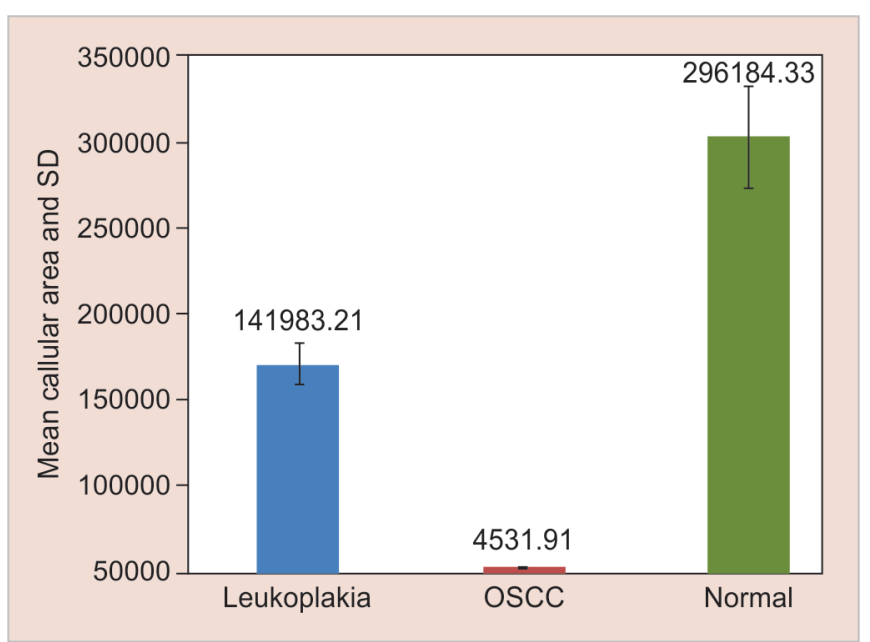

Fig. 5: Comparison of cellular area in leukoplakia, OSCC, and normal healthy subjects

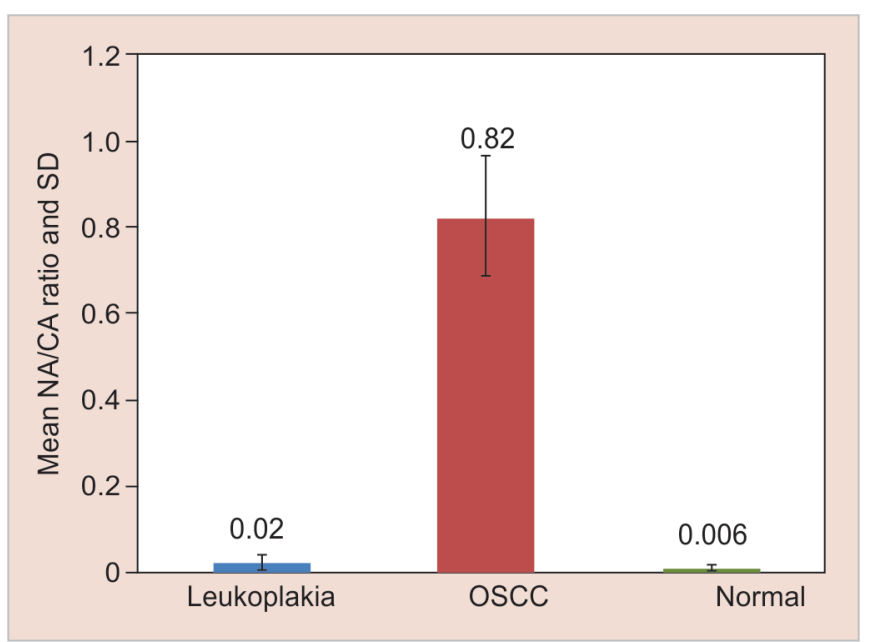

Fig. 7: Comparison of nuclear area and cellular area ratio in leukoplakia, OSCC, and normal healthy subjects 
Different parameters analyzed on intergroup comparison provided statistically significant results.

Results of the study showed an increase in ND, NA with the reduction in $C D, C A$, and alteration in N/C ratio suggesting early indicators of dysplastic or malignant changes in leukoplakia and OSCC.

\section{Discussion}

It is well documented that exfoliative cytology is used as a screening tool in the early detection of oral cancer. This method is comparatively cheaper and accurate in the early diagnosis of PMDs and oral cancer. There are certain limitations of this technique, such as, inadequate sampling, contamination, and low sensitivity.

In the present study, cytomorphometric variations of the cells in the smears were analyzed using research microscopy. The software is simple to use and convenient to analyze the smears as it provides a magnified image. It was found that the mean value of ND and NA, N/C ratio of cells was higher in OSCC, followed by the PMD. In the alteration of any cell, the fundamental defect occurs at the molecular level with a chain of reactions affecting the total cell morphology and its system. The biologic activity is reflected in the nucleus, and functional activity is reflected in the cytoplasm. ${ }^{9}$ The neoplastic epithelial cells of OSCC show a significant increase in NA attributing to the increased contents of the nucleus allowing for replication. Aneuploidy is also an early marker for dysplasia. It was in accordance with the study of Weigum et al. ${ }^{10}$ In their study of 41 cases, the smears were analyzed under a compound microscope and the mean NA of normal mucosa, dysplasia, and OSCC was 63.4 $\pm 11,149 \pm 23$, and $165 \pm 46$, respectively. The NA was significantly increased in the cytologic smears of dysplasia, and OSCC smears when compared to normal mucosa $(p<0.001)$. Similarly, in the study done by Khandelwal and Solomon, analysis of smears under a compound microscope revealed that the mean NA of cells was higher in OSCC when compared to tobacco users and normal healthy individuals. ${ }^{4}$ In another study conducted on smokers and non-smokers by Goregen et al., it was observed that there was a $16.5 \%$ rise in the NA of smokers when compared to non-smokers. ${ }^{11}$

The values of CA from the smears of normal controls, leukoplakia, and OSCC in the present study, showed a mean value of $1,535.80 \pm 79.38,1,078.51 \pm 56.65$, and $769.70 \pm 38.77 \mu^{2}$. There was a gradual decrease in CA from the normal oral mucosa, leukoplakia, and OSCC, respectively. ANOVA test for comparing the mean CA of three groups revealed significantly different values among the groups $(p<0.001)$. It was also found that the size of the cell was decreasing conversely with increasing nuclear size. With increasing activity, the maturing ability of the cytoplasm diminishes. The cell also generates less amount of cytoplasm concerning the nucleoplasm. ${ }^{4}$

The study conducted by Khandelwal and Solomon showed similar results. ${ }^{4}$ In total 60 cases, 20 cases each of normal mucosa, mucosa of tobacco users and OSCC the mean value was 1,377.30 \pm $76.68,1,120 \pm 70.44$, and $783.62 \pm 53.33$, respectively. The CA was significantly different among the groups. The study conducted by Cowpe et al. and Ogden et al. showed similar findings when the smears were analyzed under a compound microscope. ${ }^{12-14}$

The mean value of the NA/CA ratio in the present study from oral smears of normal healthy individuals, leukoplakia, and OSCC was $0.043 \pm 0.004,0.100 \pm 0.008$, and $0.181 \pm 0.015$, respectively.
There is a significantly different NA/CA ratio among the groups ( $p<$ 0.001 ) while comparing the mean NA/CA ratio of the three groups. This could be due to a significant rise in mean NA and a significant decrease in mean CA.

A similar finding was observed in the study by Cowpe et al., and Khandelwal and Solomon. In another study performed by Diniz-Freitas et al., with 10 healthy subjects and 10 patients with OSCC, analysis of smears under compound microscope reported that neither NA and CA nor NA/CA ratio differs between OSCC and healthy subjects. ${ }^{15}$ The findings are contrary, and the reason could be due to the small sample size, the difference in the methodology employed, selection of site, and the inclusion of only superficial cells. The altered ratio of squamous cells indicates less differentiation of basal and suprabasal cells. The appearance of these cells in the superficial epithelium also indicates a dysplastic change.

The basis of exfoliative cytology relies on the normal physiology of oral epithelium. Deeper layers of cells are strongly adherent in normal conditions. In the case of pathological alterations, the deeper layers become loose and exfoliate, along with normal epithelial cells. In OSCC, more of the basal and parabasal cells can be seen.

The study clearly summarizes that an increase in the NA and a decrease in the CA are the main characteristics of malignant keratinocytes. Thus, morphometric evaluation of epithelial keratinocytes can serve as a beneficial diagnostic adjunct for early screening and detection of oral cancer. Clinical diagnosis and relevant history though played a major part in considering the lesions for the study, not evaluating histopathology may add as a limitation to the study, and hence further studies with histopathology aid in supplementing the results in future studies.

\section{Conclusion}

Exfoliative cytology using a commercially available toothbrush is a simple, non-invasive, relatively painless, and inexpensive technique that could be used in the early diagnosis of PMDs and OSCC decreasing patient morbidity and improving patient survival. Quantitative parameters, such as, morphometry are reproducible and eliminate the observer bias as it is obtained by software analysis and hence improves the accurateness in the diagnosis. Brush biopsy can also be effective in mass screening of dysplasias, PMDs, and early carcinomas.

\section{References}

1. Parkin DM, Bray F, Ferlay J, et al. Global cancer statistics, 2002. CA Cancer J Clin 2005;55(2):74-108. DOI: 10.3322/canjclin.55.2.74.

2. Mehrotra R, Singh M, Kumar D, et al. Age specific incidence rate and pathological spectrum of oral cancer in Allahabad. Ind J Med Sci 2003;57:400-404.

3. Prasad LK. Burden of oral cancer: an Indian scenario. J Orofac Sci 2014;6(2):77. DOI: 10.4103/0975-8844.143043.

4. Khandelwal S, Solomon MC. Cytomorphological analysis of keratinocytes in oral smears from tobacco users and oral squamous cell carcinoma lesions. A histochemical approach. Int J Oral Sci 2010;2(1):45-52. DOI: 10.4248/IJOS10011.

5. Chitturi RT, Devy AS, Nirmal RM, et al. Oral lichen planus: a review of etiopathogenesis, clinical, histological and treatment aspects. J Interdiscipl Med Dent Sci 2014;2(05):142. DOI: 10.4172/2376032X.1000142.

6. Chitturi RT, Kumar VA, Naik P, et al. Oral submucous fibrosis - an Indian perspective. Research 2014;1:702. DOI: 10.13070/rs.en.1.702. 
7. Chitturi R, Sindhuja P, Parameswar R, et al. A clinical study on oral lichen planus with special emphasis on hyperpigmentation. J Pharm Bioallied Sci 2015;7:S495-S498. DOI: 10.4103/0975-7406. 163513.

8. Winn DM. Tobacco use and oral disease. J Dent Educ 2001;65(4):306312. DOI: 10.1002/j.0022-0337.2001.65.4.tb03400.x.

9. Sivapathasundharam B, Kalasagar M. Yet another article on exfoliative cytology. J Oral Maxillofac Pathol 2004. 54-57.

10. Weigum SE, Floriano PN, Redding SW, et al. Nano-bio-chip sensor platform for examination of oral exfoliative cytology. Cancer Prev Res (Phila) 2010;3(4):518-528. DOI: 10.1158/1940-6207.CAPR-09-0139.

11. Goregen $M$, Akgul HM, Gundogdu C. The cytomorphological analysis of buccal mucosa cells in smokers. Turk J Med Sci 2011;41:205-210.
12. Cowpe JG, Longmore RB, Green MW. Quantitative exfoliative cytology of abnormal oral mucosal smears. J R Soc Med 1988;81(9):509-513. DOI: $10.1177 / 014107688808100905$.

13. Ogden GR, Cowpe JG, Green MW. Quantitative exfoliative cytology of normal buccal mucosa: effect of smoking. J Oral Pathol Med 1990;19(2):53-55. DOI: 10.1111/j.1600-0714.1990.tb00795.x.

14. Ogden GR, Cowpe JG, Green MW. The effect of distant malignancy upon quantitative cytologic assessment of normal oral mucosa. Cancer 1990;65(3):477-480. DOI: 10.1002/1097-0142(19900201)65:3<477::AIDCNCR2820650317>3.0.CO;2-G.

15. Diniz-Freitas M, Garcia-Garcia A, Crespo-Abelleira A, et al. Applications of exfoliative cytology in the diagnosis of oral cancer. Med Oral 2004;9:355-361. 